\title{
Challenges and priorities for E-cigarette regulation at the local level - insights from an Ontario tobacco control community-of-practice
}

Ryan David Kennedy ${ }^{1,2^{*}}$, Susan Himel ${ }^{3}$, Irene Lambraki ${ }^{2}$ and Stephanie Filsinger ${ }^{2}$

\begin{abstract}
Background: There has been minimal policy development in Canada to regulate when and where e-cigarettes can be used, and no policies to-date to set a minimum legal sale age to purchase e-cigarettes. Public Health professionals that are members of an Ontario-wide Community-of-Practice (CoP) working on tobacco control issues were surveyed about e-cigarette activity at their health units.

Findings: The survey was completed by 19 respondents from 17 different health units (response rate of 63\%; representing $47 \%$ of the province's health units). When respondents were asked to describe how 'high a priority' the issue of e-cigarettes was within their health unit, $88 \%(n=15)$ reported it was a 'medium' or 'high'. The vast majority of members of the CoP $(90 \%, n=17)$ reported that their health unit is experiencing questions from the public about the safety or health risks of e-cigarettes (e.g. e-juice, nicotine cartridges, poisoning, second-hand vapour), as well as questions about the efficacy of e-cigarettes to support cessation $(90 \%, n=17)$. Almost three quarters of respondents $(74 \%, n=14)$ reported that their health unit has received complaints about people using e-cigarettes in enclosed workplaces, and roughly one quarter $(26 \%, n=5)$ reported their health unit has received complaints about outdoor e-cigarette use.
\end{abstract}

Conclusions: Most members of the CoP report that their local health unit is engaged in the issue of electronic cigarettes. Local authorities including cities and regions have the jurisdictional authority to regulate many dimensions of electronic smoking products including the creation of e-cigarette 'vapour-free' environments, and regulating sales to youth.

Keywords: E-cigarettes, Electronic cigarettes, Health units, Community of practice, Smoke-free Ontario

\section{Background}

Electronic cigarettes (e-cigarettes) are battery operated devices that deliver an aerosol to the user [1]. In 2009, Health Canada issued a warning to consumers advising against the use of e-cigarettes [2]. Health Canada also issued a notice to stakeholders indicating that any electronic smoking product that enabled a user to inhale doses of nicotine would be considered a new drug and therefore regulated by the Food and Drugs Act. Any

\footnotetext{
* Correspondence: rdkennedy@jhu.edu

'Institute for Global Tobacco Control, Department of Health, Behavior \& Society, Johns Hopkins Bloomberg School of Public Health, Baltimore, MD, USA

${ }^{2}$ Propel Centre for Population Health Impact, University of Waterloo, Waterloo, Canada

Full list of author information is available at the end of the article
}

electronic smoking product - including e-cigarettes, cigars, cigarillos and pipes, as well as cartridges of nicotine solutions and related products, requires authorization to be imported, advertised or sold in Canada [3]. To date, no electronic smoking product has been authorized for sale in Canada [4].

It is currently legal to sell e-cigarette devices in Canada that do not include nicotine, and do not make a health claim - such as 'this product will help you quit smoking'. Despite the lack of market authorization and warnings from Health Canada, e-cigarettes with and without nicotine are sold in stores [5], as well as available to order from internet retailers [6]. Reports from the NGO community explain that e-cigarettes are available for purchase in Canada in a variety of retail environments including convenience stores, gas stations, tobacconists, and 
mall kiosks [7]. A study of retailers in the Ontario East Tobacco Control Area Network (TCAN-East) found that e-cigarettes were available for purchase from $19 \%$ of licensed tobacco-retail outlets. From the TCAN-East sample, more than half the chain-convenience stores (58\%) and almost half the gas stations (44\%) sold e-cigarettes [8]. This study did not report if the products for sale contained nicotine. Products without nicotine can be sold in Canada to anyone; there is currently no age-restriction on the sale of an e-cigarette. Analysts report that Canada saw a significant rise in the marketing and sale of e-cigarettes between 2012 and 2013 [9].

Given that traditional cigarettes have such a deep impact on health, it is "virtually certain" that e-cigarettes are less harmful; however, the impact of e-cigarettes on public health is uncertain [10]. NGOs have shared concern that e-cigarettes may be 'starter' products for youth [11]. Recent work in Canada has identified that among a sample of young people (age 16-30), current and former smokers are more likely to have tried e-cigarettes compared to never smokers, although the study reported evidence of use among non-smokers [12]. Researchers from the tobacco control community worry that widespread use of e-cigarettes may erode some of the gains that have been achieved including smoke-free spaces and tobacco-use denormalization [13,14].

In Canada there is no federal, provincial/territorial, or local legislation that regulates the use of electronic cigarettes in workplaces or enclosed public places [15]. E-cigarette industry websites proclaim that their products can be used indoors or other environments where smoking is prohibited. Many airlines including Air Canada [16] and hospitality venues like Starbucks have enacted their own policies that prohibit customers from using e-cigarettes [17]. Many NGOs have provided position statements that include suggestions for e-cigarette regulation $[18,19]$. The regulatory priorities outlined in these statements include the need for policies to restrict the sale of e-cigarette products to minors, and the need to create environments where the use of e-cigarettes is not permitted [20].

In Canada, local municipalities have been pioneers developing and enacting tobacco control policies decades before provinces/territories passed similar laws. In the province of Ontario, there is extensive public infrastructure to support local by-law development including the 36 public health units that administer health promotion and disease prevention programs. Across Ontario, health unit staff are coordinated into 7 geographic Tobacco Control Area Networks (TCANs), to work collaboratively on tobacco control issues. TCANs are supported by the provincial government through mechanisms created by the Smoke-free Ontario Act (SFOA) (2006) [21]. The SFOA also supports a resource centre named the Program Training and Consultation Centre (PTCC).
The PTCC supports a Community of Practice (CoP) dedicated to Tobacco-Free Sports and Recreation and SmokeFree Outdoor Spaces (TFSR CoP). A CoP is a group of people "who share a concern or a passion for something they do and learn how to do it better as they interact regularly" [22]. Members of the TFSR CoP have regular teleconferences and meet twice a year in-person to discuss policy and programs to support tobacco control. The CoP includes members from public health research institutions, NGOs, each TCAN and 27 of 36 health units.

The present study sought to understand current activities in local health units in Ontario related to e-cigarettes. The study also sought to measure the degree to which e-cigarettes represent a priority for tobacco control staff in Ontario health units currently participating in the TFSR CoP and to identify what data or resources would help support local health units to address e-cigarettes in their communities.

\section{Research hypothesis}

The research team hypothesized that members of the TFSR CoP would be engaged in work related to e-cigarettes including providing consultation and resources to: support businesses and workplaces to develop e-cigarette-free policies, provide support to citizens asking about e-cigarettes safety and tobacco-use cessation, and answering questions from potential retailers interested in setting up an e-cigarette business.

\section{Methods}

A survey was developed by members of the authorship team, staff at the PTCC, and a colleague at the NonSmokers' Rights Association. The survey was posted to a web-based survey site. A link to the survey was sent via email to all members of the TFSR CoP on May 6th, 2014. Respondents were asked to complete the survey by May 12th, 2014.

No ethics review was conducted as this was part of an internal program setting exercise.

\section{Sample and response rate}

The survey was completed by 19 respondents who represented 17 of the 36 health units in the province. A survey was completed by at least one respondent from each of the 7 Provincial TCANs. At the time of the survey, the CoP had an active membership of 27 health units. This represents a response rate of $63 \%$; approximately half of the province's health units responded to this survey $(47 \%, \mathrm{n}=17)$.

Respondents were asked to complete the survey on behalf of their health unit - so in some cases, respondents would have needed to confer with other colleagues including health inspectors, managers, and staff who monitor the health unit's tobacco information line. 


\section{Survey questions and analysis}

Question 1 asked respondents to report 'yes' or 'no' to a range of identified questions or complaints they may be receiving at their health unit. The proportion of respondents that answered 'yes' is reported in Table 1. An open ended 'other' option was also provided and the written responses are also presented in Table 1. Question 2 asked respondents to rank 'how high a priority are e-cigarettes within your health unit?'; the response options were 'high', 'medium' or 'low'. The results are presented in Figure 1. Question 3 was an open ended question; the survey asked respondents "What do you need the most help with related to e-cigarettes right now?" A complete list of responses is included in Table 2 (responses have been edited appropriately to address the use of jargon and to ensure that respondents' identities are not disclosed).

\section{Results}

\section{Survey findings}

Question 1 was answered by all 19 respondents. The results of the survey are presented in Table 1 below.

The vast majority of members of the CoP reported that their health unit is experiencing questions about the safety or health risks of e-cigarettes (e.g. e-juice, nicotine

\section{Table 1 Reported health unit activities related to e-cigarettes}

\begin{tabular}{|c|c|}
\hline $\begin{array}{l}\text { Response Options (note: respondents selected all } \\
\text { that applied) }\end{array}$ & SAID 'YES' \\
\hline $\begin{array}{l}\text { Questions about the safety or health risks of e-cigarettes } \\
\text { (e.g. e-juice, nicotine cartridges, poisoning, second-hand } \\
\text { vapour) }\end{array}$ & $90 \%(n=17)$ \\
\hline Questions about legality or illegality of e-cigarettes & $74 \%(n=14)$ \\
\hline Questions regarding e-cigarettes as a cessation tool & $90 \%(n=17)$ \\
\hline $\begin{array}{l}\text { Complaints about sales of e-cigarettes or e-liquid } \\
\text { to youth }\end{array}$ & $47 \%(n=9)$ \\
\hline $\begin{array}{l}\text { Complaints from individuals about indoor e-cigarette } \\
\text { vaping }\end{array}$ & $74 \%(n=14)$ \\
\hline $\begin{array}{l}\text { Complaints from individuals about outdoor e-cigarette } \\
\text { vaping }\end{array}$ & $26 \%(n=5)$ \\
\hline $\begin{array}{l}\text { Calls about e-cigarettes from individual schools and/or } \\
\text { school boards }\end{array}$ & $53 \%(n=10)$ \\
\hline $\begin{array}{l}\text { Calls about e-cigarettes from colleges and/or other } \\
\text { post-secondary institutions }\end{array}$ & $32 \%(n=6)$ \\
\hline Calls about e-cigarettes from workplaces & $68 \%(n=13)$ \\
\hline Calls about e-cigarettes from local media & $32 \%(n=6)$ \\
\hline $\begin{array}{l}\text { Requests to help develop a position statement on } \\
\text { e-cigarettes }\end{array}$ & $37 \%(n=7)$ \\
\hline Requests to help develop a policy on e-cigarettes & $58 \%(n=11)$ \\
\hline Requests to include e-cigarettes under SFO by-laws & $32 \%(n=6)$ \\
\hline
\end{tabular}

"Not getting many requests from municipalities but are trying to encourage them". cartridges, poisoning, second-hand vapour), as well as questions about the efficacy of e-cigarettes to support cessation $(90 \%, \mathrm{n}=17)$. Almost three quarters of respondents $(74 \%, n=14)$ reported that their health unit has received complaints about people using e-cigarettes inside, and roughly a quarter $(26 \%, \mathrm{n}=5)$ reported they had received complaints about outdoor e-cigarette use. More than half of respondents reported that their health unit has received requests to help develop policies on e-cigarettes. More than half of respondents reported that their local school board(s) had contacted them about e-cigarettes. About a third of the respondents (32\%, $\mathrm{n}=6$ ) indicated that their health unit had received requests asking for e-cigarettes to be included under existing smoke-free by-laws.

When respondents were asked to describe how 'high a priority' e-cigarettes was within their health unit, $68 \%$ $(\mathrm{n}=13)$ reported it was a 'medium' priority, while more than one-fifth $(n=4)$ reported that it was a 'high' priority. See Figure 1 below.

Respondents were asked to identify what they needed the "most help with related to e-cigarettes right now?" Responses are reported in Table 2 below. Each response is from a unique respondent.

Most respondents $(90 \%, \mathrm{n}=17)$ provided a response to the open-ended question. Many indicated that their health unit would benefit from sample policies, including policies for workplaces or schools. Many respondents also indicated that a position statement or agreed upon speaking notes would be helpful to answer questions about product safety and/or cessation efficacy. Approximately a third of respondents requested further research to help their health unit address issues about the safety of e-cigarettes, efficacy, their appeal to youth, and the impact of 'second-hand vapour'.

\section{Conclusions and discussion}

Public Health units in Ontario are currently engaged in addressing e-cigarettes in their communities. The

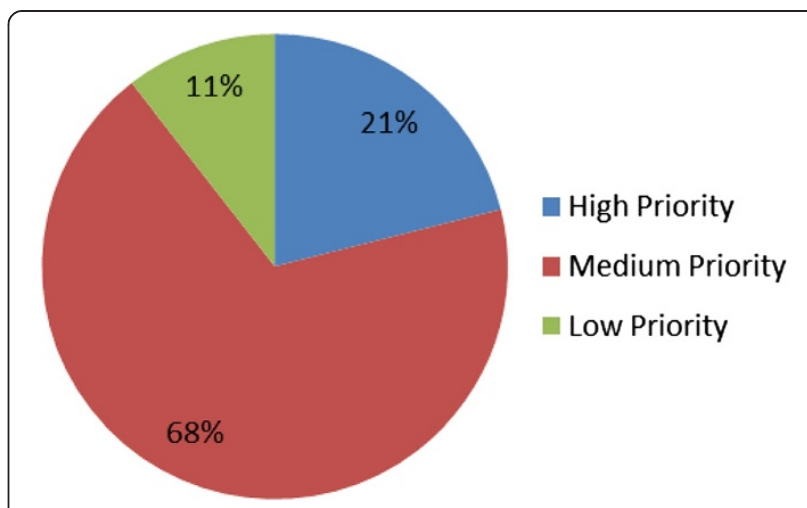

Figure 1 Reported priority of e-cigarettes in health unit. 
Table 2 Identified supports that would be helpful for health units to address e-cigarettes

Survey respondent's answers

1 Draft policies help from Health Canada to get them off the shelves.

2 Gathering literature to show [that] e-cigarettes are not harmless and that nicotine is still considered a harmful substance that can have negative physiological effects.

3 A clearer understanding of what those within the health community that support the e-cigarette are actually supporting, i.e. open sale to anyone, use in public places and workplaces, no regulation?

4 Talking points to public on why they are a risk, not a cessation tool, not covered in [Smoke-free Ontario Act] advocacy to create e-cig legislation.

5 Policy statement re: need for e-cig policy to address protection issues to be sent around to [Medical Officers of Health] (in tandem with awareness and presentation to [Canadian Medical Officers of Health] through [Association of Local Public Health Agencies] for less progressive [Public Health units] to feel comfortable adapting... E-cig key messages.. to be finalized and circulated to [Public Health units] for use. Webinar to discuss the key messages along with TCAN scan + [Non-Smokers' Rights Association] to advise of opportunities to advance the issue.

6 It's pretty hard to rate what priority level e-cigs are for the health unit when we have no official policy at this point to use to advocate with community partners. We, like many others, are waiting for some definitive research or an interim risk management decision to at least treat [the use of e-cigarettes]... like tobacco products until more is known. Ideally this would come from the province. what we need is a really good statement - consistent wording we could all use instead of each jurisdiction or agency creating its own version that may or may not stand up to scrutiny if it is tested. Ideally it would include tobacco and tobacco related or smoking/vaping devices and products and would capture water pipes, e-cigs and any new products that may come along.

7 We previously prepared a report for council in the fall of 2013 on the status of e-cigarettes. At this time we prepared a fact sheet for the public and posted it on our website. We continue to receive questions from the public and recently a Councilor has asked about e-cigarettes wondered about inclusion in smoke-free bylaws. Because of this, we are currently in the middle of writing a briefing note for our $\mathrm{MOH}$ and perhaps an updated report to council on e-cigarettes.

8 For a common, agreed upon statement from health care officials (they are divided right now and it is making policy or any other work around e-cigs difficult).

9 Sample policies for workplaces and schools. Reliable/concise info on e-cig as a cessation tool.

10 We'd like a sense of actions in other jurisdictions (Ontario, Canada and international), as it is hard to keep track of the changing policy landscape. It is also hard to keep track of the changing research landscape, so we'd like to know what existing/emerging or planned research exists on e-cigarette safety.

11 Advocacy at the provincial and federal levels for regulation and research.

12 More research and evidence about dangers of using e-cigarettes. Regulation of the product

13 A common position statement on the use of e-cigarettes.

14 We need more research on various components of e-cigarettes (cessation efficacy, appeal to youth, safety, second hand vapour, etc.)

15 Synthesis of the available (high quality) evidence on e-cigarettes.

16 Key messages sample policies.

17 Keep sending most up-to-date research and recommendations on e-cigs. majority of respondents reported that their health unit is working to address citizen concerns about the safety or health risks of e-cigarettes, as well as addressing questions about e-cigarettes efficacy to support tobacco-use cessation. Most health units are also addressing questions or concerns about second-hand e-cigarette vapour. Many respondents indicated that they would benefit from sample policies for workplaces and schools to regulate e-cigarette use. There was also an identified need for additional research to help support and inform policy at the local level. A similar study in the United States, [23] that measured priorities for local/state level policy makers, identified the need for research/evidence around the health effects from secondhand vapour exposure.

Public Health professionals understand that e-cigarettes may represent a transformative technology. There is also awareness that these products could introduce a new generation of youth to nicotine; youth may become addicted to nicotine from e-cigarettes or transition from e-cigarettes to traditional cigarettes. There is also a concern that e-cigarettes could compromise some of the tobacco control policy gains made over the last 15 years in Ontario including smoke-free spaces. Many of these potential risks could be minimized by regulation including policies that restrict the sale of e-cigarettes to youth. Local communities can address the uncertainty around risk from exposure to second-hand vapour by banning or restricting where people can use e-cigarettes. Many communities in the United States have banned the use of e-cigarettes wherever regular cigarette use is banned [24]. Currently only two communities in Canada have banned e-cigarette use in outdoor settings.

Local health units played a critical role in the development, implementation and evaluation of smoke-free policies and, given the local public health lead agency role in rolling out the SFO, they are well positioned to help communities regulate e-cigarette use and sales. Local health units are also well positioned to advocate for additional federal/provincial policies such as regulating product flavours, particularly flavours that may appeal to young children.

Evidence and co-ordination is needed to mitigate possible risks, and to ensure the possible public health gains from potentially less harmful products, such as e-cigarettes, are realized.

\section{Abbreviations}

NSRA: Non-Smokers' Rights Association; PTCC: Program Training and Consultation Centre; CoP: Community of Practice; TCANs: Tobacco Control Area Networks.

\section{Competing interests}

The authors declare that they have no competing interests.

Ryan David Kennedy is a member and co-leader of the Community of Practice (COP) that identified the need for this research; he did not participate in the survey. 
Irene Lambraki is employed by the University of Waterloo as a Senior Research Associate for a contract with PTCC. She does not have direct contact with the community of practice studied in this paper. Susan Himel is the Health Promotion Specialist, Knowledge Broker for the TFSR COP.

\section{Authors' contributions}

RDK conceptualized the paper and was the lead author. SH, RDK and MT developed the survey tool. SH organized and summarized data tables. SF supported manuscript formatting, including referencing. All authors provided comment and edits to the drafts of the manuscripts. All authors read and approved the final manuscript.

\section{Acknowledgements}

The authors wish to acknowledge the members of the Tobacco-free Sports and Recreation and Smoke-Free Outdoor Spaces Community of Practice, which is co-ordinated and supported by the Program Training and Consultation Centre - a resource centre of the Smoke Free Ontario Strategy.

The survey used in this study was developed in part by Melodie Tilson, Director of Policy, Non-Smokers' Rights Association. Additional support for the survey development was provided by Nancy Bradshaw and Jason Chapman of the PTCC. The authors thank Nancy Bradshaw and Lynn Ward for her comments on a draft of this manuscript.

Support formatting this manuscript was provided by Ornell Douglas, Propel Centre for Population Health Impact.

\section{Funding}

The Propel Centre for Population Health Impact is supported from Major Program grant from the Canadian Cancer Society Research Initiative (CCSRI grant \#701019).

\section{Author details}

${ }^{1}$ Institute for Global Tobacco Control, Department of Health, Behavior \& Society, Johns Hopkins Bloomberg School of Public Health, Baltimore, MD, USA. ${ }^{2}$ Propel Centre for Population Health Impact, University of Waterloo, Waterloo, Canada. ${ }^{3}$ PTCC (Program Training and Consultation Centre), LEARN (Learning through Evidence, Action and Reflection Networks), Toronto, Canada.

Received: 24 June 2014 Accepted: 15 October 2014 Published: 28 October 2014

\section{References}

1. FDA US Food and Drug Administration: Electronic Cigarettes (e-Cigarettes) What are electronic cigarettes? 2014. http://www.fda.gov/newsevents/ publichealthfocus/ucm172906.htm]

2. Health Canada: Health Canada Advises Canadians Not to Use Electronic Cigarettes. http://www.healthycanadians.gc.ca/recall-alert-rappel-avis/hc-sc/ 2009/13373a-eng.php.

3. Health Canada: Notice: To All Persons Interested in Importing, Advertising or Selling Electronic Smoking Products in Canada. [http://www.hc-sc.gc.ca/dhp-mps/alt_formats/pdf/prodpharma/applicdemande/pol/notice_avis_e-cig-eng.pdf.

4. Miller A: E-cigarette debate divides regulators and consumers. Can Med Assoc J 2014, 186:5.

5. Vancouver Coastal Health: E-cigarettes - harmful or helpful? 2014. https:// www.vch.ca/about_us/news/NCH-e-cigarettes.

6. Smoking and Health Action Foundation/Non-Smokers' Rights Association: Report on the Forum on E-Cigarettes. http://www.nsra-adnf.ca/cms/file/ files/SHAF_E-cig_Forum_Report_Feb_2013-FINAL.pdf.

7. Non-Smokers' Rights Association: The Buzz on E-Cigarettes. 2012. https:// www.quitnow.ca/database/files/library/e_cig_Brochure_NSRA_FINAL.pdf.

8. McKegney N, Kruz A, McWilliams D, McKercher Mortimer A, Steel O'Conner K: E-Cigarette Prevalence in Eastern Ontario. http://www.smokefreeottawa.com/ uploads/1/1/7/4/11742147/ecigarette_prevalence_in_eastern_ontario_web.pdf.

9. Euromonitor International: Tobacco in Canada" Country Report. 2013. http:// www.euromonitor.com/tobacco-in-canada/report.

10. International Tobacco Control Project: ITC Canada National Report. Findings from the Wave 1 to 8 Surveys (2002-2011). Waterloo, Ontario Canada: University of Waterloo; 2013.
11. Myers ML, Campaign for Tobacco Free Kids, Press Release: ,CDC Survey Finds Youth E-Cigarette Use More than Doubled from 2011-2012, Shows Urgent Need for FDA Regulation. http://www.tobaccofreekids.org/ press_releases/post/2013_09_05_ecigarettes.

12. Czoli CD, Hammond D, White CM: Electronic cigarettes in Canada: Prevalence of use and perceptions among youth and young adults. Can J Public Health 2014, 105(2):e97-e102.

13. Canadian Public Health Association: E-cigarettes - an update. Spring 2014, XXXVIII:1.

14. O'Loughlin JL: E-cigarettes - Promise or peril? Can J Public Health 2014 105(2):e94-e96.

15. Middlesex-London Health unit: Electronic Cigarettes (E-Cigarettes) Assessment of Evidence and Implications for Middlesex-London Health unit Recommendations. 2013. https://www.healthunit.com/uploads/ecigarettes-mlhu-position.pdf.

16. Air Canada: Information and Services. Carry-on Baggage - Accepted Items. 2014. http://www.aircanada.com/en/travelinfo/airport/baggage/ carry-on.html\#faq:5-6-*.

17. Personal communication: June 9, 2014, Starbucks Canada Customer Care Communication \#200-12114783\#.

18. International Union against Tuberculosis and Lung Disease. Oct. 2013: Position Statement on Electronic Cigarettes [ECs] or Electronic Nicotine Delivery Systems [ENDS]. Available here: http://www.theunion.org/whatwe-do/publications/official/body/E-cigarette_statement_FULL.pdf.

19. Non-Smokers' Rights Association/Smoking and Health Action Foundation. November 2013: Position Statement on Electronic Cigarettes. Available here: http://www.nsra-adnf.ca/cms/file/files/NSRA_ecig_ position_statement_final_Nov13.pdf.

20. Tobacco Legal Consortium: Regulating Electronic Cigarettes and Similar Devices. Tips \& Tools. http://publichealthlawcenter.org/sites/default/files/ resources/tclc-guide-reg-ecigarettes-2014_0.pdf.

21. Service Ontario: Smoke-free Ontario Act. 2006. http://www.e-laws.gov.on.ca/ html/regs/english/elaws_regs_060048_e.htm.

22. Wenger E: Communities of practice: Learning, meaning and identity. In 1998. Cambridge, UK: Cambridge University Press; 1998.

23. Schmitt CL, Lee YO, Curry LE, Farrelly MC, Rogers T: Research support for effective state and community tobacco control programme response to electronic nicotine delivery systems. Tob Control 2014, 23:iii54-iii57.

24. New York City Government: Electronic Cigarettes Law. 2014. http://www1. nyc.gov/nyc-resources/service/1591/electronic-cigarettes-law.

doi:10.1186/1617-9625-12-18

Cite this article as: Kennedy et al:: Challenges and priorities for E-cigarette regulation at the local level - insights from an Ontario tobacco control community-of-practice. Tobacco Induced Diseases 2014 12:18

\section{Submit your next manuscript to BioMed Central and take full advantage of:}

- Convenient online submission

- Thorough peer review

- No space constraints or color figure charges

- Immediate publication on acceptance

- Inclusion in PubMed, CAS, Scopus and Google Scholar

- Research which is freely available for redistribution 